Bull. Soc. math. France

129 (1), 2001, p. 141-157

\title{
ÉTALE COHOMOLOGY AND REDUCTION OF ABELIAN VARIETIES
}

\author{
By A. Silverberg \& Yu.G. Zarhin
}

\begin{abstract}
In this paper we study the étale cohomology groups associated to abelian varieties. We obtain necessary and sufficient conditions for an abelian variety to have semistable reduction (or purely additive reduction which becomes semistable over a quadratic extension) in terms of the action of the absolute inertia group on the étale cohomology groups with finite coefficients.

RÉSumÉ (Cohomologie étale et réduction des variétés abéliennes)

Nous étudions les groupes de cohomologies étales associés aux variétés abéliennes. Nous obtenons des conditions nécessaires et suffisantes pour qu'une variété abeliénne ait une réduction semistable (ou une réduction purement additive qui devient semistable sur une extension quadratique), en termes de l'action du groupe d'inertie absolu sur les groupes de cohomologies étales à coefficients finis.
\end{abstract}

\section{Introduction}

Suppose $X$ is a smooth projective variety over a field $F, v$ is a discrete valuation on $F$, and $\ell$ is a prime number not equal to the residue characteristic of $v$. Let $F^{s}$ denote a separable closure of $F$, let $\bar{v}$ be an extension of $v$ to $F^{s}$,

Texte reçu le 15 novembre 1999

A. Silverberg, Mathematics Department, Ohio State University, Columbus, Ohio (USA). E-mail : silver@math.ohio-state.edu

Yu.G. Zarhin, Mathematics Department, Pennsylvania State University, University Park, PA (USA) and Institute for Mathematical Problems in Biology, Russian Academy of Sciences, Pushchino, Moscow Region (Russia). • E-mail : zarhin@math.psu.edu

2000 Mathematics Subject Classification. - 11G10.

Key words and phrases. - Abelian varieties, semistable reduction, étale cohomology, monodromy. 
let $I$ denote the inertia subgroup at $\bar{v}$ of $\operatorname{Gal}\left(F^{s} / F\right)$, and let $\bar{X}=X \times_{F} F^{s}$. For every positive integer $k$, the group $I$ acts naturally on the $k$-th $\ell$-adic étale cohomology group $H_{\text {ét }}^{k}\left(\bar{X}, \mathbb{Q}_{\ell}\right)$. Grothendieck proved the Monodromy Theorem (see the Appendix to [10], and 1.2 and 1.3 of [4]), which says that $I$ acts on $H_{\text {êt }}^{k}\left(\bar{X}, \mathbb{Q}_{\ell}\right)$ via quasi-unipotent operators, i.e., for every $\sigma \in I$ we have $\left(\sigma^{m}-1\right)^{r} H_{\mathrm{èt}}^{k}\left(\bar{X}, \mathbb{Q}_{\ell}\right)=0$ for some positive integers $m$ and $r$. It is known (see 3.7 of [4], and 3.5 and 3.6 of [5]) that if $k=1$, then one may take $r=2$. It easily follows (see Theorem 5.6 (i), (ii) below) that if $X$ is an abelian variety, then one may take $r=k+1$. It is shown in [6] (see 3.4 and 3.8 of [4], and p. vi of [3]) that one may take $r=k+1$ whenever one knows the Purity Conjecture (3.1 of [4]) and resolution of singularities.

From now on, suppose $X$ is a $d$-dimensional abelian variety. The Néron model $\mathcal{X}$ of $X$ at $v$ is a smooth separated model of $X$ over the valuation ring $R$ such that for every smooth scheme $\mathcal{Y}$ over $R$ and morphism $\varphi: \mathcal{Y} \otimes_{R} F \rightarrow X$ over $F$ there is a unique morphism $\mathcal{Y} \rightarrow \mathcal{X}$ over $R$ which extends $\varphi$. The generic fiber of $\mathcal{X}$ can be canonically identified with $X$, and $\mathcal{X}$ is a commutative group scheme over $R$ whose group structure extends that of $X$. Let $X_{v}^{0}$ denote the identity component of the special fiber of $\mathcal{X}$ at $v$. Over an algebraic closure of the residue field, there is an exact sequence of algebraic groups

$$
0 \rightarrow U \times \mathcal{T} \longrightarrow X_{v}^{0} \longrightarrow B \rightarrow 0
$$

where $B$ is an abelian variety, $\mathcal{T}$ is the maximal algebraic torus in $X_{v}^{0}$, and $U$ is a unipotent group. By definition, $X$ is semistable at $v$ if and only if $U=0$. As $I$ modules, $H_{\text {ét }}^{1}\left(\bar{X}, \mathbb{Z}_{\ell}\right) \cong \operatorname{Hom}_{\mathbb{Z}_{\ell}}\left(T_{\ell}(X), \mathbb{Z}_{\ell}\right)$, where $T_{\ell}(X)$ is the $\ell$-adic Tate module. Grothendieck's Galois Criterion for Semistability says that $X$ is semistable at $v$ if and only if every $\sigma \in I$ acts on $T_{\ell}(X)$ as a unipotent operator of echelon $\leq 2$, i.e., if and only if $(\sigma-1)^{2} H_{\text {ét }}^{1}\left(\bar{X}, \mathbb{Z}_{\ell}\right)=0$ for every $\sigma \in I$.

Suppose $n$ is a positive integer relatively prime to the residue characteristic of $v$. Raynaud's criterion says that if $I$ acts trivially on the $n$-torsion $X_{n}$, and $n \geq 3$, then $X$ is semistable at $v$. The authors (see [13] and [15]) proved that if $n \geq 5$, then $X$ is semistable at $v$ if and only if $(\sigma-1)^{2} X_{n}=0$ for every $\sigma \in I$. In other words, necessary and sufficient conditions for semistability can be read off not only from the $\ell$-adic representation, as shown by Grothendieck, but also from the mod $n$ representation (for $n \geq 5$ ). The aim of this paper (see Theorem 5.10) is to generalize this result to the case of the higher étale cohomology groups $H^{k}$. Note that $H_{\text {ét }}^{1}(\bar{X}, \mathbb{Z} / n \mathbb{Z})$ and $X_{n}$ are dual. Assume that $0<k<2 d$, that $k<r \in \mathbb{Z}$, and that $n$ does not belong to a certain finite set $N(r)$ of prime powers, defined explicitly in terms of $r$ in $\S 2$. (For example, $N(2)=\{1,2,3,4\}$.) We show that if $k$ is odd, then $X$ is semistable at $v$ if and only if $(\sigma-1)^{r} H_{\text {êt }}^{k}(\bar{X}, \mathbb{Z} / n \mathbb{Z})=0$ for every $\sigma \in I$. If $k$ is even, we show (under an additional assumption; see $\S 7$ ) that $(\sigma-1)^{r} H_{\mathrm{et}}^{k}(\bar{X}, \mathbb{Z} / n \mathbb{Z})=0$ for every $\sigma \in I$ if and only if either $X$ is semistable at $v$ or $X$ has purely additive 
reduction at $v$ but is semistable over a (ramified) quadratic extension of $F$. We call the latter abelian varieties briefly unstable (see Definitions 5.2 and 5.3).

We gave such necessary and sufficient conditions for semistability in the case $k=1$ and $r=2$ in [13] and [15]. Poincaré duality then gives such criteria in the case $k=2 d-1$. One can check that in the cases $k=1$ or $2 d-1$, one cannot replace $N(r)$ by a smaller set. However, when $2 \leq k \leq 2 d-2$ one may replace $N(r)$ by an explicitly defined subset $N^{\prime}(r)$ for which the result is sharp (see $\S 6)$.

In $\S 2$ we introduce basic definitions and notation. Section 3 deals with multilinear algebra in characteristics 0 and $\ell$ and over $\mathbb{Z}_{\ell}$. We use the Jordan decompositions of exterior powers of linear operators to obtain a MinkowskiSerre type result. Section 4 contains some abelian variety results that will be used later. In $\S \S 5-6$ we state and prove our main results. We give necessary and sufficient conditions for semistability, and also necessary and sufficient conditions for an abelian variety to either be semistable or have purely additive reduction which becomes semistable over a quadratic extension. In $\S 6$ we shrink the exceptional set in the criteria when $2 \leq k \leq 2 d-2$. We prove that this exceptional set is minimal.

We hope that our results and/or methods will be useful in the study of semistability for the more general class of motives [9].

Silverberg would like to thank IHES, the Bunting Institute, and the Mathematics Institute of the University of Erlangen-Nürnberg for their hospitality, and NSA, NSF, the Science Scholars Fellowship Program at the Bunting Institute, and the Alexander von Humboldt-Stiftung for financial support. Zarhin would like to thank NSF and Université Paris-Sud for financial support and Centre Émile Borel (Institut Henri Poincaré) and University of Glasgow for their hospitality. He also would like to thank Ed Formanek for helpful discussions.

\section{Notation and definitions}

If $F$ is a field, let $F^{s}$ denote a separable closure. Throughout this paper, $X$ is a $d$-dimensional abelian variety defined over $F$, and $v$ is a discrete valuation on $F$ of residue characteristic $p \geq 0$. Let $\bar{v}$ denote an extension of $v$ to $F^{s}$, and let $I$ denote the inertia subgroup at $\bar{v}$ of $\operatorname{Gal}\left(F^{s} / F\right)$. If $\ell$ is a prime not equal to the characteristic of $F$, let

$$
\rho_{\ell, X}: \operatorname{Gal}\left(F^{s} / F\right) \longrightarrow \operatorname{Aut}\left(T_{\ell}(X)\right) \cong \mathrm{GL}_{2 d}\left(\mathbb{Z}_{\ell}\right)
$$

denote the $\ell$-adic representation on the Tate module $T_{\ell}(X)=\lim X_{\ell^{n}}$. Let $V_{\ell}(X)=T_{\ell}(X) \otimes_{\mathbb{Z}_{\ell}} \mathbb{Q}_{\ell}$, let $\bar{X}=X \times_{F} F^{s}$, and let

$$
H_{\ell}^{k}=H_{\mathrm{et}}^{k}\left(\bar{X}, \mathbb{Z}_{\ell}\right)=\operatorname{Hom}_{\mathbb{Z}_{\ell}}\left(\wedge^{k}\left(T_{\ell}(X)\right), \mathbb{Z}_{\ell}\right) .
$$

BULletin DE LA SOCiÉtÉ MATHÉMATIQUE DE FRANCE 
Then $H_{\ell}^{k}$ is a free $\mathbb{Z}_{\ell}$-module of $\operatorname{rank}\left(\begin{array}{c}2 d \\ k\end{array}\right)=: b$, and $H_{\ell}^{k} \otimes_{\mathbb{Z}_{\ell}} \mathbb{Q}_{\ell}=\wedge_{\mathbb{Q}_{\ell}}^{k}\left(V^{*}\right)$, where $V^{*}=\operatorname{Hom}_{\mathbb{Q}_{\ell}}\left(V, \mathbb{Q}_{\ell}\right)$ is the dual vector space. Let

$$
\rho_{\ell, k}: I \longrightarrow \operatorname{Aut}\left(H_{\ell}^{k}\right) \cong \mathrm{GL}_{b}\left(\mathbb{Z}_{\ell}\right)
$$

be the representation giving the action of $I$ on $H_{\ell}^{k}$.

Definition 2.1. - If $r$ is a positive integer, define a finite set of prime powers $N(r)$ by

$$
N(r)=\left\{\text { prime powers } \ell^{m} \mid 0 \leq m(\ell-1) \leq r\right\} .
$$

For example, $N(1)=\{1,2\}, N(2)=\{1,2,3,4\}, N(3)=\{1,2,3,4,8\}$, $N(4)=\{1,2,3,4,5,8,9,16\}$.

\section{Some linear algebra}

Lemma 3.1. - Suppose $g$ is a linear operator on a finite-dimensional vector space over a field of characteristic zero. Suppose $\left(g^{m}-1\right)^{2}=0$ for some positive integer $m$. If $g$ is unipotent, then $(g-1)^{2}=0$. If $-g$ is unipotent, then $(g+1)^{2}=0$.

Proof. - If $g$ is unipotent then all the eigenvalues of $g^{m-1}+\cdots+g+1$ are $m \neq 0$. Therefore $g^{m-1}+\cdots+g+1$ is an invertible operator. Since

$$
0=\left(g^{m}-1\right)^{2}=(g-1)^{2}\left(g^{m-1}+\cdots+g+1\right)^{2}
$$

we have $(g-1)^{2}=0$.

If $-g$ is unipotent then all the eigenvalues of $g^{m}$ are $(-1)^{m}$. Since $g^{m}$ is unipotent, $m=2 r$ is even. We have

$$
0=\left(g^{m}-1\right)^{2}=\left(g^{2 r}-1\right)^{2}=(g+1)^{2}(g-1)^{2}\left(g^{2(r-1)}+\cdots+g^{2}+1\right)^{2} .
$$

All the eigenvalues of $g-1$ are -2 and all the eigenvalues of $g^{2(r-1)}+\cdots+g^{2}+1$ are $r \neq 0$. Therefore $g-1$ and $g^{2(r-1)}+\cdots+g^{2}+1$ are invertible, and $(g+1)^{2}=0$.

THEOREM 3.2. - Suppose $m, r$ and $g$ are positive integers, $\ell$ is a prime number, and $A \in \mathrm{M}_{g}\left(\mathbb{Z}_{\ell}\right)$ satisfies $(A-1)^{r} \in \ell^{m} \mathrm{M}_{g}\left(\mathbb{Z}_{\ell}\right)$. Suppose $\lambda$ is an eigenvalue of $A$ which is a root of unity. If $r=m(\ell-1)$ then $\lambda^{\ell}=1$. If $r<m(\ell-1)$ then $\lambda=1$.

Proof. - This is a special case of Theorem 6.7 of [12].

TheOREM 3.3. - Suppose $\overline{\mathbb{Z}}$ is the ring of algebraic integers in $\overline{\mathbb{Q}}, n$ and $r$ are positive integers, $\lambda$ is a root of unity in $\overline{\mathbb{Z}}$, and $(\lambda-1)^{r} \in n \overline{\mathbb{Z}}$. Then $\lambda=1$ if $n \notin N(r)$.

Proof. - This is a special case of Corollary 3.3 of [12].

TOME $129-2001-\mathrm{N}^{\mathrm{O}} 1$ 
LEMMA 3.4 (see Lemma 4.3 of [13]). - Suppose $\mathcal{O}$ is an integral domain of characteristic zero, and $\ell$ is a prime number. Suppose $r, s$, and $m$ are positive integers such that $r \geq m \ell^{s-1}(\ell-1)$. Suppose $\alpha \in \mathcal{O}$ and $\alpha^{\ell^{s}}=1$. Then $(\alpha-1)^{r} \in \ell^{m} \mathbb{Z}[\alpha]$.

Suppose $V$ is a finite-dimensional vector space over $\mathbb{Q}_{\ell}$. Suppose $k$ is an integer and $0<k<\operatorname{dim}(V)$. Let

$$
f_{k}: \mathrm{GL}(V) \longrightarrow \mathrm{GL}\left(\wedge^{k}(V)\right)
$$

denote the natural representation defined by $f_{k}(g)=\wedge^{k}(g)$. For $g \in \operatorname{GL}(V)$, let $g=s_{g} u_{g}=u_{g} s_{g}$ be the Jordan decomposition, where $u_{g}, s_{g} \in \mathrm{GL}(V), u_{g}$ is unipotent, and $s_{g}$ is semisimple. Then $f_{k}(g)=f_{k}\left(s_{g}\right) f_{k}\left(u_{g}\right)=f_{k}\left(u_{g}\right) f_{k}\left(s_{g}\right)$, $f_{k}\left(u_{g}\right)$ is unipotent, and $f_{k}\left(s_{g}\right)$ is semisimple.

REMARK 3.5. - In the notation of $\S 2$, with "** denoting the dual map, for every $\sigma \in I$ we have

$$
\rho_{\ell, k}(\sigma)=\left(f_{k}\left(\rho_{\ell, X}\left(\sigma^{-1}\right)\right)\right)^{*} .
$$

REMARK 3.6. - The kernel of $f_{k}$ is $\left\{\gamma \in \mathbb{Z}_{\ell} \mid \gamma^{k}=1\right\}$. In particular, if $(k, \ell-1)=1$ then $f_{k}$ is injective.

LEMMA 3.7. - The element $\wedge^{k}(g)$ is unipotent if and only if there exists a $k$-th root of unity $\gamma \in \mathbb{Z}_{\ell}$ such that $\gamma g$ is unipotent.

Proof. - If $\wedge^{k}(g)$ is unipotent, then $\wedge^{k}\left(s_{g}\right)=1$. By Remark 3.6, $s_{g}$ is a $k$-th root of unity in $\mathbb{Z}_{\ell}$. Let $\gamma=s_{g}^{-1}$.

Now assume that $V$ is even-dimensional, choose a non-degenerate alternating bilinear form on $V$, and let $\operatorname{Sp}(V) \subset \mathrm{GL}(V)$ be the corresponding symplectic group. Write

$$
\rho_{k}: \operatorname{Sp}(V) \longrightarrow \mathrm{GL}\left(\wedge^{k}(V)\right)
$$

for the restriction of $f_{k}$ to $\operatorname{Sp}(V)$. It is well-known that $u_{g}, s_{g} \in \operatorname{Sp}(V)$ for every $g \in \operatorname{Sp}(V)$.

REMARK 3.8. - Remark 3.6 easily implies that the kernel of $\rho_{k}$ is $\{1\}$ if $k$ is odd and is $\{1,-1\}$ if $k$ is even.

Lemma 3.9. - Suppose that $g \in \operatorname{Sp}(V)$.

(i) If $(g-1)^{2}=0$, then $\left(\rho_{k}(g)-1\right)^{k+1}=0$.

(ii) If $k$ is even and $(g+1)^{2}=0$, then $\left(\rho_{k}(g)-1\right)^{k+1}=0$.

(iii) If $\gamma g$ is unipotent for some $\gamma \in \mathbb{Q}_{\ell}$, then $\gamma \in\{ \pm 1\}$.

(iv) Suppose $\wedge^{k}(g)$ is unipotent. If $k$ is odd then $g$ is unipotent. If $k$ is even then either $g$ or $-g$ is unipotent.

BULLETIN DE LA SOCIÉtÉ MATHÉMATIQUE DE FRANCE 
Proof. - Note that

$$
\left(\rho_{k}(g)-1\right)\left(v_{1} \wedge v_{2} \wedge \cdots \wedge v_{k}\right)=g v_{1} \wedge \cdots \wedge g v_{k}-v_{1} \wedge v_{2} \cdots \wedge v_{k} .
$$

Part (i) follows by substituting $g=1+\eta$, and (ii) follows from (i) applied to $-g$. Suppose now that $g$ is not unipotent. Then $g$ has an eigenvalue $\lambda \neq 1$. Since $g \in \operatorname{Sp}(V), \lambda^{-1}$ is also an eigenvalue of $g$. Suppose also that $\gamma g$ is unipotent for some $\gamma \in \mathbb{Q}_{\ell}$. Then $\gamma \lambda$ and $\gamma / \lambda$ are eigenvalues of the unipotent element $\gamma g$ and thus are equal to 1 . Therefore $\gamma^{2}=1$, i.e., $\gamma \in\{ \pm 1\}$, so either $g$ or $-g$ is unipotent. If $\wedge^{k}(g)$ is unipotent, then $\gamma g$ is unipotent for some $\gamma \in \mathbb{Q}_{\ell}$, by Lemma 3.7. If $k$ is odd then $\wedge^{k}(-g)=-\wedge^{k}(g)$ is not unipotent, so $-g$ is not unipotent and thus $g$ is unipotent.

Theorem 3.12 below will be used in $\S 6$. To prove it, we first prove a lemma and a theorem.

LEMma 3.10. - Suppose $\mathcal{W}$ is an $(\ell-1)$-dimensional vector space over a field of prime characteristic $\ell \geq 5$, and $A$ is a unipotent linear operator on $\mathcal{W}$ whose Jordan form consists of one Jordan block of size $\ell-1$. If $r \in \mathbb{Z}$ and $2 \leq r \leq \ell-3$, then $\left(\wedge^{r}(A)-1\right)^{\ell-1} \neq 0$.

Proof. - It follows immediately from Corollary III.2.7(a) on p. 43 and Proposition III.2.10(b) on p. 45 of [1] that all but one of the Jordan blocks of $\wedge^{r}(A)$ have size $\ell$, and the size of the remaining block is 1 or $\ell-1$ (since the size is less than $\ell$ and is congruent $\bmod \ell$ to $\left.\operatorname{dim}\left(\wedge^{r}(\mathcal{W})\right)=\left(\begin{array}{c}\ell-1 \\ r\end{array}\right) \equiv(-1)^{r}\right)$. Since $\ell \geq 5$ and $2 \leq r \leq \ell-3$, we have $\operatorname{dim}\left(\wedge^{r}(\mathcal{W})\right) \geq \ell$. Therefore, $\wedge^{r}(A)$ must have a Jordan block of size $\ell$, so $\left(\wedge^{r}(A)-1\right)^{\ell-1} \neq 0$.

THEOREM 3.11. - Suppose $\mathcal{V}$ is a finite-dimensional vector space over a field of characteristic $\ell \geq 5$, and $A$ is a unipotent linear operator on $\mathcal{V}$. Suppose $k \in \mathbb{Z}, 2 \leq k \leq \operatorname{dim}(\mathcal{V})-2$, and $(A-1)^{\ell-2} \neq 0$. Then $\left(\wedge^{k}(A)-1\right)^{\ell-1} \neq 0$.

Proof. - Let $\mathcal{W}$ be an $A$-invariant $(\ell-1)$-dimensional subspace of $\mathcal{V}$ such that the Jordan form of the restriction of $A$ to $\mathcal{W}$ is a Jordan block of size $\ell-1$. Then $\wedge^{j}(\mathcal{W})$ is a $\wedge^{j}(A)$-invariant subspace of $\wedge^{j}(\mathcal{V})$, for all $j$. By Lemma 3.10,

$$
\left(\wedge^{j}(A)-1\right)^{\ell-1}\left(\wedge^{j}(\mathcal{W})\right) \neq 0
$$

if $2 \leq j \leq \ell-3$. We are therefore done if $k \leq \ell-3$. Suppose $k>\ell-3$. Then $\wedge^{k-(\ell-3)}(\mathcal{V} / \mathcal{W}) \neq 0$. Since $A$ is unipotent, $\wedge^{k-(\ell-3)}(A)$ is also unipotent, and there exists a non-zero $\wedge^{k-(\ell-3)}(A)$-invariant element $u \in \wedge^{k-(\ell-3)}(\mathcal{V} / \mathcal{W})$. Then

$$
\begin{aligned}
0 \neq\left(\wedge^{\ell-3}(A)-1\right)^{\ell-1}\left(\wedge^{\ell-3}(\mathcal{W})\right) \cong\left(\wedge^{\ell-3}(A)-1\right)^{\ell-1}\left(\wedge^{\ell-3}(\mathcal{W})\right) \otimes u \\
=\left(\wedge^{k}(A)-1\right)^{\ell-1}\left(\wedge^{\ell-3}(\mathcal{W}) \otimes u\right) \\
\quad \subset\left(\wedge^{k}(A)-1\right)^{\ell-1}\left(\wedge^{\ell-3}(\mathcal{W}) \otimes \wedge^{k-(\ell-3)}(\mathcal{V} / \mathcal{W})\right)
\end{aligned}
$$

TOME $129-2001-\mathrm{N}^{\mathrm{O}} 1$ 
There is a $\wedge^{k}(A)$-equivariant projection from the image in $\wedge^{k}(\mathcal{V})$ of

$$
\wedge^{\ell-3}(\mathcal{W}) \otimes \wedge^{k-(\ell-3)}(\mathcal{V})
$$

onto $\wedge^{\ell-3}(\mathcal{W}) \otimes \wedge^{k-(\ell-3)}(\mathcal{V} / \mathcal{W})$. Therefore, $0 \neq\left(\wedge^{k}(A)-1\right)^{\ell-1}\left(\wedge^{k}(\mathcal{V})\right)$.

Theorem 3.12. - Suppose $\ell$ is a prime number, $\ell \geq 5, V$ is a finite-dimensional $\mathbb{Q}_{\ell}$-vector space, $T$ is a $\mathbb{Z}_{\ell}$-lattice in $V$, and $g$ is a quasi-unipotent linear operator on $V$ such that $g(T)=T$. Suppose $k$ and $m$ are positive integers and $2 \leq k \leq \operatorname{dim}(V)-2$. If $\left(\wedge^{k}(g)-1\right)^{m(\ell-1)} \in \ell^{m} \operatorname{End}\left(\wedge^{k}(T)\right)$, then $\wedge^{k}(g)$ is unipotent.

Proof. - By Theorem 3.2, all the eigenvalues of $\wedge^{k}(g)$ are $\ell$-th roots of unity. In particular, $\wedge^{k}(g)^{\ell}$ is unipotent. By Lemma 3.7 there exists a $k$-th root of unity $\gamma \in \mathbb{Z}_{\ell}$ such that $(\gamma g)^{\ell}$ is unipotent. Replacing $g$ by $\gamma g$, we may assume that $g^{\ell}$ is unipotent.

First, suppose that $g$ is semisimple. Then $g^{\ell}=1$ and $\wedge^{k}(g)^{\ell}=1$. By Theorem 6.8 of [12] there is a $\wedge^{k}(g)$-invariant splitting of the free $\mathbb{Z}_{\ell}$-module $\wedge^{k}(T)$ into a direct sum of free $\mathbb{Z}_{\ell}$-modules $\wedge^{k}(T)=P_{1} \oplus P_{2}$ such that $\wedge^{k}(g)$ acts as the identity on $P_{1}$, and

$$
\wedge^{k}(g)^{\ell-1}+\cdots+\wedge^{k}(g)+1=0 \quad \text { on } \quad P_{2} .
$$

This implies easily that

$$
\left(\wedge^{k}(g)-1\right)^{\ell-1} \in \ell \operatorname{End}\left(\wedge^{k}(T)\right) .
$$

If $g \neq 1$ then $g$ has an eigenvalue which is a primitive $\ell$-th root of unity, and therefore by Theorem $3.2,(g-1)^{\ell-2} \notin \ell \operatorname{End}(T)$. If we let $\mathcal{V}=T / \ell T$, and let $A: \mathcal{V} \rightarrow \mathcal{V}$ be the linear operator induced by $g$, then $(A-1)^{\ell-2} \neq 0$, but $\left(\wedge^{k}(A)-1\right)^{\ell-1}=0$. This contradicts Theorem 3.11, and proves that $g=1$ when $g$ is semisimple.

Next we will induct on the maximum of the multiplicities of the roots of the minimal polynomial $P(t)$ of $g$ (i.e., on the maximal size of the Jordan blocks for $g$ ). Let $P_{1}(t) \in \mathbb{Z}_{\ell}[t]$ be the monic polynomial whose roots are the same as those of $P(t)$, but all with multiplicity one. Then $P_{1}$ divides $P$, and $P_{1}=P$ if and only if $g$ is semisimple. Let

$$
T_{0}=\left\{x \in T \mid P_{1}(g)(x)=0\right\} .
$$

Then $T_{0}$ is a pure free $\mathbb{Z}_{\ell}$-submodule of $T$ which is $g$-invariant, and the restriction $g_{0}: T_{0} \rightarrow T_{0}$ is semisimple. Let $T_{1}=T / T_{0}$ and let $g_{1}$ denote the induced automorphism $g_{1}: T_{1} \rightarrow T_{1}$. Then $T_{1}$ is a free $\mathbb{Z}_{\ell}$-module of finite rank, and the maximal multiplicity of a root of the minimal polynomial of

$$
g_{0} \oplus g_{1}: T_{0} \oplus T_{1} \longrightarrow T_{0} \oplus T_{1}
$$

is strictly less than that of $P(t)$, if $g$ is not semisimple. Note that $\left(g_{0} \oplus g_{1}\right)^{\ell}$ is unipotent, since $g^{\ell}$ is unipotent. Further, $g_{0} \oplus g_{1}$ is unipotent if and only if $g$ 
is unipotent. To apply induction and finish the proof, it suffices to check that

$$
\left(\wedge^{k}\left(g_{0} \oplus g_{1}\right)-1\right)^{m(\ell-1)} \in \ell^{m} \operatorname{End}\left(\wedge^{k}\left(T_{0} \oplus T_{1}\right)\right) .
$$

For $0 \leq i \leq k$, let $H_{i}$ be the image of the natural homomorphism

$$
\wedge^{i}\left(T_{0}\right) \otimes \wedge^{k-i}(T) \longrightarrow \wedge^{k}(T) .
$$

Then

$$
H_{i} / H_{i+1} \cong \wedge^{i}\left(T_{0}\right) \otimes \wedge^{k-i}\left(T_{1}\right)
$$

and

$$
\wedge^{k}(T)=H_{0} \supset H_{1} \supset \cdots \supset H_{k}=\wedge^{k}\left(T_{0}\right) \supset H_{k+1}:=0
$$

is a natural filtration of $\wedge^{k}(g)$-stable pure $\mathbb{Z}_{\ell}$-submodules of $\wedge^{k}(T)$. Since $H_{i}$ is pure in $\wedge^{k}(T)$, we have $H_{i} \cap \ell^{m} \wedge^{k}(T)=\ell^{m} H_{i}$. Therefore,

$$
\left(\wedge^{k}(g)-1\right)^{m(\ell-1)}\left(H_{i}\right) \subseteq \ell^{m} H_{i} .
$$

Since

we have (1).

$$
\bigoplus_{i=0}^{k}\left(H_{i} / H_{i+1}\right)=\bigoplus_{i=0}^{k}\left(\wedge^{i}\left(T_{0}\right) \otimes \wedge^{k-i}\left(T_{1}\right)\right)=\wedge^{k}\left(T_{0} \oplus T_{1}\right),
$$

\section{Abelian variety lemmas}

As stated earlier, we suppose $X$ is an abelian variety over a field $F, v$ is a discrete valuation on $F$ of residue characteristic $p \geq 0$, and $\ell$ is a prime different from $p$. Recall that $I$ is the inertia subgroup at $\bar{v}$ of $\operatorname{Gal}\left(F^{s} / F\right)$.

Theorem 4.1 (Galois Criterion for Semistability). - The following are equivalent:

(i) $X$ is semistable at $v$;

(ii) $I$ acts unipotently on $T_{\ell}(X)$, i.e. all the eigenvalues of $\rho_{\ell, X}(\sigma)$ are 1 for every $\sigma \in I$

(iii) for every $\sigma \in I,\left(\rho_{\ell, X}(\sigma)-1\right)^{2}=0$.

Proof. — See 3.5 and 3.8 of [5], and Theorem 6 on p. 184 of [2].

LEMmA 4.2. - Suppose $\sigma \in I$. Then:

(i) $\rho_{\ell, X}(\sigma)$ is unipotent if and only if $\left(\rho_{\ell, X}(\sigma)-1\right)^{2}=0$;

(ii) $-\rho_{\ell, X}(\sigma)$ is unipotent if and only if $\left(\rho_{\ell, X}(\sigma)+1\right)^{2}=0$.

Proof. - There exists a finite Galois extension $L \subset F^{s}$ of $F$ such that if $w$ is the restriction of $\bar{v}$ to $L$ then $X$ is semistable at $w$ (see Prop. 3.6 of [5]). Let $I_{w}=I \cap \operatorname{Gal}\left(F^{s} / L\right)$ be the corresponding inertia group, let $m=[L: F]$, and let $g=\rho_{\ell, X}(\sigma)$. Then $\sigma^{m} \in I_{w}$. By Theorem 4.1, $\left(g^{m}-1\right)^{2}=0$. Now apply Lemma 3.1.

TOME $129-2001-\mathrm{N}^{\mathrm{O}} 1$ 
The following result follows immediately from Lemmas 3.9 (iii) and 4.2.

Proposition 4.3. - Suppose $\sigma \in I$. The following are equivalent:

(i) there exists $\gamma_{\sigma} \in \mathbb{Q}_{\ell}$ such that $\gamma_{\sigma} \rho_{\ell, X}(\sigma)$ is unipotent;

(ii) either $\rho_{\ell, X}(\sigma)$ or $-\rho_{\ell, X}(\sigma)$ is unipotent;

(iii) either $\left(\rho_{\ell, X}(\sigma)-1\right)^{2}=0$ or $\left(\rho_{\ell, X}(\sigma)+1\right)^{2}=0$.

Proposition 4.4. - Let $J \subset I$ denote the first ramification group, and let $\tau$ be a lift to I of a topological generator of the procyclic group $I / J$. The following are equivalent:

(i) $X$ has purely additive reduction at $v$;

(ii) 1 is not an eigenvalue for the action of $\tau$ on $V_{\ell}(X)^{J}$;

(iii) $V_{\ell}(X)^{I}=0$.

Proof. - The equivalence of (ii) and (iii) is obvious. For the equivalence of (i) and (ii) see Corollary 1.10 of [7].

\section{Higher cohomology groups of abelian varieties}

Write $V=V_{\ell}(X)$ and $T=T_{\ell}(X)$, and recall that $H_{\ell}^{k}=H_{\text {êt }}^{k}\left(\bar{X}, \mathbb{Z}_{\ell}\right)$. The image of $\rho_{\ell, X}$ lies in the symplectic group $\operatorname{Sp}(V)$, by the Galois-equivariance of the Weil pairing, and the fact that the inertia group acts as the identity on the $\ell$-power roots of unity.

Assumption 5.1. - For the remainder of this paper (except for Remark 7.1) we will assume that if $p=2$ then the valuation ring is henselian.

Definition 5.2. - If $p \neq 2$ then we say that $X$ is briefly unstable at $v$ if $X$ is purely additive at $v$ and becomes semistable above $v$ over a quadratic separable extension of $F$.

Definition 5.3. - If $p=2$ then we say that $X$ is briefly unstable at $v$ if $X$ is purely additive at $v$ and there exists a finite unramified extension $M$ of $F$ such that $X$ is semistable above $v$ over a quadratic separable extension of $M$.

Remark 5.4. - By Theorem 4.1, the quadratic extension in Definitions 5.2 and 5.3 is ramified over $v$.

Theorem 5.5. - Suppose $X$ is an abelian variety over a field $F, v$ is a discrete valuation on $F$ of residue characteristic $p \geq 0$, and $\ell$ is a prime different from $p$. Then the following are equivalent:

(a) $X$ is either semistable or briefly unstable at $v$;

(b) for each $\sigma \in I$, either $\rho_{\ell, X}(\sigma)$ or $-\rho_{\ell, X}(\sigma)$ is unipotent.

BULletin DE LA SOCiÉtÉ MATHÉMATIQUE DE FRANCE 
Proof. - Assume (a) holds. By Theorem 4.1, we may reduce to the case where $X$ has purely additive reduction at $v, M$ is a finite unramified extension of $F$, and $L$ is a quadratic separable extension of $M$ over which $X$ is semistable above $v$. Then by Theorem 4.1, $\rho_{\ell, X}(\sigma)^{2}$ is unipotent for all $\sigma \in I$. Let $J \subset I$ be the first ramification subgroup. Then $J$, and therefore $\rho_{\ell, X}(J)$, is either trivial (if $p=0$ ) or a pro-p-group. By [7] (see pp. 282-283), since $\ell \neq p, \rho_{\ell, X}(J)$ is either trivial or a finite $p$-group. If $s \in \rho_{\ell, X}(J) \subset \rho_{\ell, X}(I)$, then $s^{2}$ is unipotent and has finite order, and thus $s^{2}=1$. It follows that either $\rho_{\ell, X}(J)=\{1\}$, or $p=2$ and $\rho_{\ell, X}(J)$ is a finite commutative group of exponent 2 .

Suppose that $\rho_{\ell, X}(J)=\{1\}$. Then $V^{J}=V$. Let $\tau$ be a lift to $I$ of a topological generator of the procyclic group $I / J$. Then $g:=\rho_{\ell, X}(\tau)$ generates the procyclic group $\rho_{\ell, X}(I)$. By Prop. 4.4, 1 is not an eigenvalue of $g$. Since $g^{2}$ is unipotent, the only eigenvalue of $g$ is -1 , i.e., $-g$ is unipotent. For each integer $i$ either $g^{i}$ or $-g^{i}$ is unipotent. Since in the $\ell$-adic topology the set of integral powers of $g$ is dense in $\rho_{\ell, X}(I)$ and the set of unipotent operators in $\operatorname{Aut}(T)$ is closed, therefore for each $\sigma \in I$ either $\rho_{\ell, X}(\sigma)$ or $-\rho_{\ell, X}(\sigma)$ is unipotent.

We may thus assume that $\rho_{\ell, X}(J) \neq\{1\}, p=2$, and $\rho_{\ell, X}(J)$ is a finite commutative group of exponent 2 . We may assume that $L \subset F^{s}$. Let $w$ be the restriction of $\bar{v}$ to $L$. Let $I_{w}$ denote the inertia subgroup at $\bar{v}$ of $\operatorname{Gal}\left(F^{s} / L\right)$. Clearly, $J_{w}:=J \cap I_{w}$ is the first ramification subgroup of $I_{w}$, and $J_{w}$ has index 2 in $J$. Since $X$ is semistable at $w, \rho_{\ell, X}(\sigma)$ is unipotent for all $\sigma \in I_{w}$. Since $\rho_{\ell, X}(J)$ is finite, $\rho_{\ell, X}(\sigma)=1$ for all $\sigma \in J_{w}$. Since $J_{w}$ has index 2 in $J$, therefore $\rho_{\ell, X}(J)$ has order 2 .

Since $L / F$ is wild quadratic, therefore the inclusion $I_{w} \subset I$ induces a natural isomorphism $I_{w} / J_{w}=I / J$. Let $\tau_{w}$ be a lift to $I_{w}$ of a topological generator of $I_{w} / J_{w}=I / J$. Since $\rho_{\ell, X}\left(\tau_{w}\right)$ is unipotent, and $X$ has purely additive reduction at $v$, therefore $V^{J}=0$ by Proposition 4.4 .

Clearly $J_{w}$ is normal in $I$, since it is the intersection of normal subgroups. We can view $\rho_{\ell, X}$ as a homomorphism from $I / J_{w}$ to $\operatorname{Aut}(T)$. The image of

$$
I_{w} \longrightarrow I_{w} / J_{w} \subset I / J_{w}=I / J \times I / I_{w}
$$

is $I / J \times\{1\}$. Therefore $\rho_{\ell, X}(I / J \times\{1\})$ consists of unipotent operators. Let $s$ be the non-trivial element of $I / I_{w}$ and let $h=\rho_{\ell, X}(1 \times s)$. Then $h^{2}=1$. If $h=1$ then $\rho_{\ell, X}\left(I / J_{w}\right)$ consists of unipotent operators, so $X$ is semistable at $v$, which is not the case. So $h \neq 1$. If $h=-1$ then $\rho_{\ell, X}\left(I / J_{w}\right)$ is the union of $\rho_{\ell, X}(I / J \times\{1\})$ and $-\rho_{\ell, X}(I / J \times\{1\})$. Therefore for each $g \in \rho_{\ell, X}\left(I / J_{w}\right)$, either $g$ or $-g$ is unipotent. So we have reduced to the case where $h \neq \pm 1$. But then $V^{J}$, the eigenspace of $h$ corresponding to the eigenvalue 1 , is non-zero. This contradiction proves that (a) implies (b).

To prove that (b) implies (a), suppose that $X$ is not semistable at $v$, and suppose that for each $\sigma \in I$ either $\rho_{\ell, X}(\sigma)$ or $-\rho_{\ell, X}(\sigma)$ is unipotent. By Theorem 4.1, $\rho_{\ell, X}(\sigma)$ is not unipotent for some $\sigma \in I$. For such a $\sigma$, the

TOME $129-2001-\mathrm{N}^{\mathrm{O}} 1$ 
eigenvalues of $\rho_{\ell, X}(\sigma)$ are all -1 . Thus $V^{I}=0$. By Prop. 4.4, $X$ has purely additive reduction at $v$. Let

$$
I_{v, X}=\left\{\sigma \in I \mid \rho_{\ell, X}(\sigma) \text { is unipotent }\right\} .
$$

Then $I_{v, X} \neq I$. It is known (see pp. 354-355 of [5] and $\S 4$ of [14]) that $I_{v, X}$ is an open normal subgroup of finite index in $I$. Since $\rho_{\ell, X}\left(I_{v, X}\right)$ consists of unipotent operators, $V^{I_{v, X}} \neq 0$ by a theorem of Kolchin (p. 35 of [8]). The restriction map $\rho^{\prime}: I \rightarrow \operatorname{Aut}\left(V^{I_{v, X}}\right)$ factors through the finite group $I / I_{v, X}$. Therefore the image of $\rho^{\prime}$ is finite. If $\sigma \in I-I_{v, X}$, then $-\rho^{\prime}(\sigma)$ is unipotent and of finite order, and thus $\rho^{\prime}(\sigma)=-1$ on $V^{I_{v, X}}$. Therefore $\rho^{\prime}$ has kernel $I_{v, X}$ and image $\{ \pm 1\}$, so $\left[I: I_{v, X}\right]=2$.

First assume $p \neq 2$. Then $I$ has exactly one subgroup of index 2 and thus this subgroup must be $I_{v, X}$. Let $L / F$ be a ramified separable quadratic extension. We may assume that $L \subset F^{s}$. Let $w$ be the restriction of $\bar{v}$ to $L$. The corresponding inertia group $I_{w} \subset \operatorname{Gal}\left(F^{s} / L\right)$ has index 2 in $I$ and therefore is $I_{v, X}$. By Theorem 4.1, $X$ is semistable at $w$.

Now assume that $p=2$. Let $F^{\mathrm{ur}}=\left(F^{s}\right)^{I}$, the maximal extension of $F$ unramified above $v$. The valuation ring of $F^{\mathrm{ur}}$ is henselian and the residue field is separably closed. Let $L$ be the quadratic extension of $F^{\mathrm{ur}}$ corresponding to $I_{v, X} \subset I \cong \operatorname{Gal}\left(F^{s} / F^{\mathrm{ur}}\right)$. Then $L=F^{\mathrm{ur}}(\sqrt{c})$ for some $c \in F^{\mathrm{ur}}, F(c)$ is unramified above $v$ over $F$, and $X$ is semistable over the ramified quadratic extension $F(\sqrt{c})$ of $F(c)$.

THEOREm 5.6. - Suppose $X$ is an abelian variety over a field $F$, suppose $v$ is a discrete valuation on $F$ of residue characteristic $p \geq 0$, suppose $k$ is a positive integer, suppose $k<2 \operatorname{dim}(X)$, and suppose $\ell$ is a prime number not equal to $p$.

(i) If $k$ is odd then the following are equivalent:
(a) $X$ is semistable at $v$,
(b) for each $\sigma \in I, \rho_{\ell, k}(\sigma)$ is unipotent,
(c) for each $\sigma \in I,\left(\rho_{\ell, k}(\sigma)-1\right)^{k+1}=0$.

(ii) If $k$ is even then the following are equivalent:
(a) $X$ is either semistable or briefly unstable at $v$,
(b) for each $\sigma \in I, \rho_{\ell, k}(\sigma)$ is unipotent,
(c) for each $\sigma \in I,\left(\rho_{\ell, k}(\sigma)-1\right)^{k+1}=0$.

(iii) If $k$ is odd then the following are equivalent:

(a) $X$ is either semistable or briefly unstable at $v$,

(b) for each $\sigma \in I$, either $\rho_{\ell, k}(\sigma)$ or $-\rho_{\ell, k}(\sigma)$ is unipotent,

(c) for each $\sigma \in I$, either $\left(\rho_{\ell, k}(\sigma)-1\right)^{k+1}=0$ or $\left(\rho_{\ell, k}(\sigma)+1\right)^{k+1}=0$.

Proof. - Clearly, (c) implies (b). That (a) implies (c) follows from Remark 3.5, combined with Theorem 4.1 and Lemmas 4.2 (i) and 3.9 (i) for (i), with Theorem 5.5 and Lemma 3.9 (ii) for (ii), and with Theorem 5.5 and Lemmas 4.2 and 
3.9 (i) for (iii). Suppose we have (b). To conclude (a), apply Lemma 3.9 (iv) and Remark 3.5, combined with Theorem 5.5 for (ii) and (iii) and with Theorem 4.1 for (i).

Corollary 5.7. - Suppose $X$ is an abelian variety over a field $F$, and $v$ is a discrete valuation on $F$ of residue characteristic $p \geq 0$. Suppose $k$ and $r$ are positive integers, $k<2 \operatorname{dim}(X)$, and $k<r$. Suppose $\sigma \in I$.

(i) If either $X$ is semistable at $v$, or $k$ is even and $X$ is briefly unstable at $v$, then

for every prime $\ell \neq p$, and

$$
(\sigma-1)^{r} H_{\text {ét }}^{k}\left(\bar{X}, \mathbb{Z}_{\ell}\right)=0
$$

$$
(\sigma-1)^{r} H_{\text {ét }}^{k}(\bar{X}, \mathbb{Z} / n \mathbb{Z})=0
$$

for every positive integer $n$ not divisible by $p$.

(ii) If $k$ is odd and $X$ is briefly unstable at $v$, then for every prime $\ell \neq p$, either

$$
(\sigma-1)^{r} H_{\text {ét }}^{k}\left(\bar{X}, \mathbb{Z}_{\ell}\right)=0 \quad \text { or } \quad(\sigma+1)^{r} H_{\text {ét }}^{k}\left(\bar{X}, \mathbb{Z}_{\ell}\right)=0,
$$

and for every positive integer $n$ not divisible by $p$, either

$$
(\sigma-1)^{r} H_{\text {ét }}^{k}(\bar{X}, \mathbb{Z} / n \mathbb{Z})=0 \quad \text { or } \quad(\sigma+1)^{r} H_{\text {ét }}^{k}(\bar{X}, \mathbb{Z} / n \mathbb{Z})=0 .
$$

Proof. — The first parts follow from Theorem 5.6, since $r \geq k+1$. The second parts follow from the first parts for all prime divisors $\ell$ of $n$, since for all $i$,

$$
H_{\text {ét }}^{k}\left(\bar{X}, \mathbb{Z} / \ell^{i} \mathbb{Z}\right)=H_{\text {ét }}^{k}\left(\bar{X}, \mathbb{Z}_{\ell}\right) \otimes \mathbb{Z} / \ell^{i} \mathbb{Z} .
$$

THEOREM 5.8. - Suppose $X$ is an abelian variety over a field $F$, and $v$ is a discrete valuation on $F$ of residue characteristic $p \geq 0$. Suppose $k, n$, and $r$ are positive integers, $k<2 \operatorname{dim}(X), n$ is not divisible by $p$, and $n \notin N(r)$.

(i) Suppose that $(\sigma-1)^{r} H_{\text {ét }}^{k}(\bar{X}, \mathbb{Z} / n \mathbb{Z})=0$ for all $\sigma \in I$. Then either $X$ is semistable at $v$, or $k$ is even and $X$ is briefly unstable at $v$.

(ii) Suppose $k$ is odd, and suppose that for each $\sigma \in I$ either

$$
(\sigma-1)^{r} H_{\text {ét }}^{k}(\bar{X}, \mathbb{Z} / n \mathbb{Z})=0 \quad \text { or } \quad(\sigma+1)^{r} H_{\text {ét }}^{k}(\bar{X}, \mathbb{Z} / n \mathbb{Z})=0 .
$$

Then either $X$ is semistable at $v$, or $X$ is briefly unstable at $v$.

Proof. - Recall ([5], Thm. 4.3) that the characteristic polynomial of $\rho_{\ell, X}(\sigma)$ has integer coefficients and does not depend on the choice of $\ell \neq p$. Therefore the characteristic polynomial $P_{\sigma}$ of $\left(\rho_{\ell, k}(\sigma)-1\right)^{r} / n$ has coefficients in $\mathbb{Z}[1 / n]$ and does not depend on the choice of $\ell \neq p$. Suppose that

$$
(\sigma-1)^{r} H_{\text {ét }}^{k}(\bar{X}, \mathbb{Z} / n \mathbb{Z})=0 .
$$

Then for all prime divisors $\ell$ of $n,\left(\rho_{\ell, k}(\sigma)-1\right)^{r} H_{\ell}^{k} \subseteq n H_{\ell}^{k}$, so $P_{\sigma}$ has coefficients in $\mathbb{Z}_{\ell}$. Thus $P_{\sigma}$ has integer coefficients. Since $X$ is semistable over a finite separable extension of $F$, by Theorem 4.1, Lemma 3.9 (i), (ii), and Theorem 5.6

TOME $129-2001-\mathrm{N}^{\mathrm{O}} 1$ 
there is a positive integer $m$ such that $\left(\rho_{\ell, k}\left(\sigma^{m}\right)-1\right)^{k+1}=0$. Let $\alpha$ be an eigenvalue of $\rho_{\ell, k}(\sigma)$. Then $\left(\alpha^{m}-1\right)^{k+1}=0$, so $\alpha^{m}=1$. Since $(\alpha-1)^{r} / n$ is an eigenvalue of $\left(\rho_{\ell, k}(\sigma)-1\right)^{r} / n$ and therefore is a root of $P_{\sigma}$, it is an algebraic integer. Since $n \notin N(r)$, we have $\alpha=1$ by Theorem 3.3. Thus $\rho_{\ell, k}(\sigma)$ is unipotent. Applying Theorem 5.6, we have (i). To obtain (ii), replace -1 by +1 in the above argument.

Next we specialize Theorem 5.8i to the case $r=1$. Note that when $k=1$ we recover Raynaud's criterion for semistability (Prop 4.7 of [5]).

Theorem 5.9. - Suppose $X$ is an abelian variety over a field $F, v$ is a discrete valuation on $F, k$ and $n$ are integers, $0<k<2 \operatorname{dim}(X), n \geq 3, n$ is not divisible by the residue characteristic, and $I$ acts as the identity on $H_{\mathrm{e} \mathrm{t}}^{k}(\bar{X}, \mathbb{Z} / n \mathbb{Z})$. Then either $X$ is semistable at $v$, or $k$ is even and $X$ is briefly unstable at $v$.

The next result is an immediate corollary of Theorems 5.8 and 5.6 and Corollary 5.7.

TheOREm 5.10. - Suppose $X$ is an abelian variety over a field $F$, suppose $v$ is a discrete valuation on $F$ of residue characteristic $p \geq 0$, suppose $k, n$, and $r$ are positive integers, and suppose $\ell$ is a prime number. Suppose $k<2 \operatorname{dim}(X)$, suppose $k<r$, suppose $n \ell$ is not divisible by $p$, and suppose $n \notin N(r)$.

(i) If $k$ is odd, then the following are equivalent:

(a) $X$ is semistable at $v$,

(b) for each $\sigma \in I,(\sigma-1)^{r} H_{\text {ét }}^{k}\left(\bar{X}, \mathbb{Z}_{\ell}\right)=0$,

(c) for each $\sigma \in I,(\sigma-1)^{r} H_{\mathrm{ett}}^{k}(\bar{X}, \mathbb{Z} / n \mathbb{Z})=0$.

(ii) If $k$ is even then the following are equivalent:

(a) $X$ is either semistable or briefly unstable at $v$,

(b) for each $\sigma \in I,(\sigma-1)^{r} H_{\text {êt }}^{k}\left(\bar{X}, \mathbb{Z}_{\ell}\right)=0$,

(c) for each $\sigma \in I,(\sigma-1)^{r} H_{\text {êt }}^{k}(\bar{X}, \mathbb{Z} / n \mathbb{Z})=0$.

(iii) If $k$ is odd then the following are equivalent:

(a) $X$ is either semistable or briefly unstable at $v$,

(b) for each $\sigma \in I$ either $(\sigma-1)^{r}$ or $(\sigma+1)^{r}$ kills $H_{\mathrm{e} t}^{k}\left(\bar{X}, \mathbb{Z}_{\ell}\right)$.

(c) for each $\sigma \in I$ either $(\sigma-1)^{r}$ or $(\sigma+1)^{r}$ kills $H_{\mathrm{e} t}^{k}(\bar{X}, \mathbb{Z} / n \mathbb{Z})$.

\section{Non-extremal cohomology groups}

The results of $\S 5$ are sharp when $k=1$ or $2 d-1$. In this section we obtain sharp results when $1<k<2 d-1$. Let

$$
N^{\prime}(r)=\left\{\ell^{m} \mid 0 \leq m(\ell-1)<r \text {, or } \ell=2 \text { or } 3 \text { and } r=m(\ell-1)\right\} .
$$

Clearly, $N^{\prime}(r) \subseteq N(r)$, and a prime power $\ell^{m}$ lies in $N(r)-N^{\prime}(r)$ if and only if $\ell \geq 5$ and $r=m(\ell-1)$. 
THEOREM 6.1. - Suppose $X$ is an abelian variety over a field $F$, suppose $v$ is a discrete valuation on $F$ of residue characteristic $p \geq 0$, suppose $k$ and $r$ are positive integers, and suppose $n$ is a positive integer which is not divisible by $p$. Suppose $2 \leq k \leq 2 \operatorname{dim}(X)-2$, suppose $k<r$, and suppose $n \notin N^{\prime}(r)$.

(i) If $k$ is odd, then the following are equivalent:

(a) $X$ is semistable at $v$,

(b) for every $\sigma \in I,(\sigma-1)^{r} H_{\text {ét }}^{k}(\bar{X}, \mathbb{Z} / n \mathbb{Z})=0$.

(ii) If $k$ is even then the following are equivalent:

(a) $X$ is either semistable or briefly unstable at $v$,

(b) for every $\sigma \in I,(\sigma-1)^{r} H_{\text {ét }}^{k}(\bar{X}, \mathbb{Z} / n \mathbb{Z})=0$.

(iii) If $k$ is odd then the following are equivalent:

(a) $X$ is either semistable or briefly unstable at $v$,

(b) for each $\sigma \in I$ either $(\sigma-1)^{r}$ or $(\sigma+1)^{r}$ kills $H_{\text {ét }}^{k}(\bar{X}, \mathbb{Z} / n \mathbb{Z})$.

Proof. - If $n \notin N(r)$ then the assertion is contained in Theorem 5.10. Thus we may assume that $n=\ell^{m}$ with $\ell \geq 5$ and $r=m(\ell-1)$. If (a) holds, then (b) follows from Corollary 5.7. If (b) holds, then (a) follows from Theorems 3.12 and 5.6.

The following example shows that the condition $n \notin N^{\prime}(r)$ is sharp.

ExAmPle 6.2. - Suppose that $F$ is a discrete valuation field, $m$ and $r$ are positive integers, $\ell$ is a prime number, and $n=\ell^{m}$. Suppose that either $m(\ell-1)<r$, or $\ell=2$ and $r=m=m(\ell-1)$, or $\ell=3$ and $r=2 m=m(\ell-1)$. Let $X_{1}, Y$ be abelian varieties of positive dimension with good reduction over $F$, and such that $Y$ has an automorphism of exact order $\ell$. If $\ell \leq 3$, assume $\operatorname{dim}(Y)=1$. Let $L$ be a totally ramified degree $\ell$ extension of $F$, and let $X_{2}$ be the $L / F$-form of $Y$ corresponding to a character $\operatorname{Gal}(L / F) \hookrightarrow \operatorname{Aut}(Y)$. Then $X=X_{1} \times X_{2}$ has neither semistable nor purely additive reduction. However, by Lemma 3.4 we have $\left(\rho_{\ell, k}(\sigma)-1\right)^{r} \in n \operatorname{End}\left(H_{\ell}^{k}\right)$ for every $\sigma \in I$.

\section{Semistability over quadratic extensions}

REMARK 7.1. - When the valuation ring is henselian, then $v$ extends uniquely to $F^{s}, I$ is normal in $\operatorname{Gal}\left(F^{s} / F\right)$, and the field $\left(F^{s}\right)^{I}$ is the (unique) maximal extension of $F$ unramified over $v$. Note that Assumption 5.1 can be dropped in Theorems $5.6(\mathrm{i}), 5.10$ (i), and 6.1 (i), in Corollary 5.7 (i) if $X$ is semistable, and in Theorems 5.8 (i) and 5.9 when $k$ is odd, since it is not used. (Assumption 5.1 is only used when we deal with brief instability.)

Lemma 7.2. - Suppose $X$ is an abelian variety over a field $F, v$ is a discrete valuation on $F$ of residue characteristic $p \geq 0$, and the valuation ring is henselian. Suppose there exists a finite unramified extension $M$ of $F$ such that $X$ is semistable above $v$ over a quadratic separable extension $L$ of $M$. Suppose

TOME $129-2001-\mathrm{N}^{\mathrm{O}} 1$ 
that if $p=2$, then the residue field either is separably closed or is algebraic over $\mathbb{F}_{2}$ (e.g., is finite). Then $X$ is semistable above $v$ over a quadratic separable extension of $F$.

Proof. - If $K$ is a subfield of $F^{s}$, let $I_{K}$ denote the inertia subgroup of $I$ corresponding to $K$. Note that $I_{L}$ is an open subgroup of $I$ of index 2 .

When $p \neq 2$, then $F$ has a tamely ramified separable quadratic extension $L^{\prime}$. Since $p \neq 2, I$ has exactly one open subgroup of index 2 , so $I_{L}=I_{L^{\prime}}$. By Theorem 4.1 over $L$ and over $L^{\prime}, X$ is semistable above $v$ over $L^{\prime}$.

Now suppose $p=2$.

If the residue field is separably closed, then $F$ has no non-trivial unramified extensions, so $M=F$.

Suppose the residue field $k$ is algebraic over $\mathbb{F}_{2}$, and let $G_{k}:=\operatorname{Gal}\left(k^{s} / k\right)$. Then $G_{k}$ is a torsion-free procyclic group, since it is a closed subgroup of $\hat{\mathbb{Z}}$. Let $F^{\mathrm{ur}}$ be the maximal unramified extension of $F$. Then $I=\operatorname{Gal}\left(F^{s} / F^{\mathrm{ur}}\right)$. Since the valuation ring is henselian, $G_{k}=\operatorname{Gal}\left(F^{\mathrm{ur}} / F\right)$. We may assume that $X$ is not semistable at $v$. Let $I_{v, X}$ be the group defined by formula (2) (proof of Theorem 5.5) with $\ell=3$. Applying Theorem 4.1 over $F$ and over $L$ (for $\ell=3$ ) shows that $I_{v, X}$ is a proper subgroup of $I$ and $I_{L} \subseteq I_{v, X} \subset I$. Since $\left[I: I_{L}\right]=2$, we have $I_{v, X}=I_{L}$ and $\left[I: I_{v, X}\right]=2$. Let $F^{\prime}$ be the quadratic extension of $F^{\text {ur }}$ cut out by $I_{v, X}$. Then $F^{\prime} / F$ is Galois, since the group $I_{v, X}$ is the intersection of $I$ and

$$
\left\{\sigma \in \operatorname{Gal}\left(F^{s} / F\right) \mid \rho_{3, X}(\sigma) \text { is unipotent }\right\},
$$

and both are stable under conjugation by $\operatorname{Gal}\left(F^{s} / F\right)$. Further, $X$ is semistable (above $v$ ) over $F^{\prime}$ by Theorem 4.1 , and $G:=\operatorname{Gal}\left(F^{\prime} / F\right)$ is an extension of $G_{k}$ by $C:=\operatorname{Gal}\left(F^{\prime} / F^{\mathrm{ur}}\right)$. This extension is central, since $C$ has order 2 and thus has no non-trivial automorphisms. Since every group whose quotient by its center is (pro)cyclic must be commutative, $G$ is commutative. Let $\Delta$ be the subset of squares in $G$. Then $\Delta$ is a closed subgroup, $\Delta \cap C=1$, and $[G: \Delta]=2$ or $4\left(G_{k}\right.$ is procyclic, so $2 G_{k}$ has index 1 or 2 in $\left.G_{k}\right)$. Thus $\Delta$ is open and $G / \Delta$ either has order 2 (in which case let $H=\Delta$ ) or is isomorphic to $\mathbb{Z} / 2 \mathbb{Z} \times \mathbb{Z} / 2 \mathbb{Z}$ and we may choose the isomorphism so that the first factor is the image of $C$ (and then let $H$ be the preimage in $G$ of the second factor). Then $H$ is an open subgroup of $G$ of index 2 , so the corresponding subfield $L^{\prime}:=\left(F^{\prime}\right)^{H}$ is quadratic over $F$. Since $H \cap C=1$, therefore $F^{\prime} / L^{\prime}$ is unramified and so $X$ is semistable over $L^{\prime}$.

Corollary 7.3. - Suppose that if the residue characteristic is 2 , then the residue field either is separably closed or is algebraic over $\mathbb{F}_{2}$. Then we may replace "briefly unstable at $v$ " by "purely additive at $v$ and becomes semistable above $v$ over a quadratic separable extension of $F$ " in the results of $\S \S 5-6$. 
REMARK 7.4. - The proof of Lemma 7.2 shows that the condition that the residue field $k$ be algebraic over $\mathbb{F}_{2}$ can be replaced by the condition that $\mathrm{Gal}\left(k^{s} / k\right)$ be a torsion-free procyclic group.

REMARK 7.5. - The group $I_{v, X}$ defined in (2) is independent of $\ell$ (see p. 355 of [5] and Theorem 4.2 of [14]). It follows that for each fixed $\sigma \in I$, whether or not $(\sigma-1)^{r}$ kills $H_{\text {ét }}^{k}\left(\bar{X}, \mathbb{Z}_{\ell}\right)$ (or $H_{\text {ét }}^{k}(\bar{X}, \mathbb{Z} / n \mathbb{Z})$ for $n$ not a power of 2 ) is independent of $\ell$ (and $n$ ), and depends only on whether or not $\sigma \in I_{v, X}$.

\section{BIBLIOGRAPHY}

[1] Almkvist (G.), Fossum (R.) - Decomposition of exterior and symmetric powers of indecomposable $\mathbb{Z} / p \mathbb{Z}$-modules in characteristic $p$ and relations to invariants, in Séminaire d'Algèbre Paul Dubreil 1976/r7n (M.P. Malliavin, ed.), Lecture Notes in Math., vol. 641, Springer, BerlinHeidelberg-New York, 1978, pp. 1-111.

[2] Bosch (S.), Lütkebohmert (W.), Raynaud (M.) - Néron models, Springer, Berlin-Heidelberg-New York, 1990.

[3] Deligne (P.) - Introduction to [6], pp. v-vii.

[4] Deligne (P.) - Résumé des premiers exposés de A. Grothendieck, in [6], pp. 1-24.

[5] Grothendieck (A.) - Modèles de Néron et monodromie, in [6], pp. 313523.

[6] Grothendieck (A.) (ed.) - Groupes de monodromie en géométrie algébrique, SGA7 I, Lecture Notes in Math., vol. 288, Springer, BerlinHeidelberg-New York, 1972.

[7] Lenstra JR. (H.W.), OORT (F.) - Abelian varieties having purely additive reduction, J. Pure and Applied Algebra, t. 36 (1985), pp. 281-298.

[8] SERRE (J.-P.) - Lie algebras and Lie groups, Second edition, Lecture Notes in Math., vol. 1500, Springer, Berlin, 1992.

[9] SerRe (J.-P.) - Propriétés conjecturales des groupes de Galois motiviques et des représentations $\ell$-adiques, in Motives (U. Jannsen, S. Kleiman, J.-P. Serre, eds.), Proc. Symp. Pure Math. 55, Part 1, AMS, Providence, 1994, pp. 377-400.

[10] Serre (J.-P.), TAte (J.) - Good reduction of abelian varieties, Ann. of Math., t. 88 (1968), pp. 492-517.

[11] Silverberg (A.), ZArhin (Yu.G.) - Semistable reduction and torsion subgroups of abelian varieties, Ann. Inst. Fourier, t. 45-2 (1995), pp. 403420.

[12] Silverberg (A.), Zarhin (YU.G.) - Variations on a theme of Minkowski and Serre, J. Pure and Applied Algebra, t. 111 (1996), pp. 285302.

TOME $129-2001-\mathrm{N}^{\mathrm{O}} 1$ 
[13] Silverberg (A.), Zarhin (Yu.G.) - Semistable reduction of abelian varieties over extensions of small degree, J. Pure Applied Algebra, t. 132 (1998), pp. 179-193.

[14] Silverberg (A.), Zarhin (Yu.G.) - Subgroups of inertia groups arising from abelian varieties, J. Algebra, t. 209 (1998), pp. 94-107.

[15] Silverberg (A.), Zarhin (YU.G.) - Reduction of abelian varieties, to appear in the Proceedings of the NATO/CRM Conference on the Arithmetic and Geometry of Algebraic Cycles. 